\title{
Electric double layer effect on observable characteristics of the tunnel current through a bridged electrochemical contact
}

\author{
Kuznetsov, A.M.; Medvedev, I.G.; Ulstrup, Jens
}

Published in:

Journal of Chemical Physics

Link to article, DOI:

$10.1063 / 1.2766954$

Publication date:

2007

Document Version

Publisher's PDF, also known as Version of record

Link back to DTU Orbit

Citation (APA):

Kuznetsov, A. M., Medvedev, I. G., \& Ulstrup, J. (2007). Electric double layer effect on observable characteristics of the tunnel current through a bridged electrochemical contact. Journal of Chemical Physics, 127(10), 104708. https://doi.org/10.1063/1.2766954

\section{General rights}

Copyright and moral rights for the publications made accessible in the public portal are retained by the authors and/or other copyright owners and it is a condition of accessing publications that users recognise and abide by the legal requirements associated with these rights.

- Users may download and print one copy of any publication from the public portal for the purpose of private study or research.

- You may not further distribute the material or use it for any profit-making activity or commercial gain

- You may freely distribute the URL identifying the publication in the public portal 


\title{
Electric double layer effect on observable characteristics of the tunnel current through a bridged electrochemical contact
}

\author{
Alexander M. Kuznetsov ${ }^{\text {a) }}$ and Igor G. Medvedev \\ A.N. Frumkin Institute of Physical Chemistry and Electrochemistry, Russian Academy of Science, \\ Leninskii prospect 31, 119991 Moscow, Russian Federation \\ Jens Ulstrup \\ Technical University of Denmark, Institute of Chemistry, Bldg. 207, DK-2800 Lyngby, Denmark
}

(Received 26 April 2007; accepted 3 July 2007; published online 13 September 2007)

\begin{abstract}
Scanning tunneling microscopy and electrical conductivity of redox molecules in conducting media (aqueous or other media) acquire increasing importance both as novel single-molecule science and with a view on molecular scale functional elements. Such configurations require full and independent electrochemical potential control of both electrodes involved. We provide here a general formalism for the electric current through a redox group in an electrochemical tunnel contact. The formalism applies broadly in the limits of both weak and strong coupling of the redox group with the enclosing metal electrodes. Simple approximate expressions better suited for experimental data analysis are also derived. Particular attention is given to the effects of the Debye screening of the electric potential in the narrow tunneling gap based on the limit of the linearized Poisson-Boltzmann equation. The current/overpotential relation shows a maximum at a position which depends on the ionic strength. It is shown, in particular, that the dependence of the maximum position on the bias voltage may be nonmonotonous. Approximate expressions for the limiting value of the slope of the current/overpotential dependence and the width of the maximum on the bias voltage are also given and found to depend strongly on both the Debye screening and the position of the redox group in the tunnel gap, with diagnostic value in experimental data analysis. (C) 2007 American Institute of Physics. [DOI: 10.1063/1.2766954]
\end{abstract}

\section{INTRODUCTION}

Electron tunneling through molecular bridge contacts in condensed matter environment (in situ) has come to attract increasing attention. Perspectives include single-molecule electronics and chemical processes and their possible role in new molecular scale electronics. In situ tunneling through redox molecules with low-lying accessible electronic levels inserted between the substrate and tip electrodes in electrochemical in situ scanning tunneling microscopy (STM) or between a pair of electrochemically controlled nanogap electrodes offer particular perspectives. The redox level(s) can thus bring the molecule to display rectification, amplification, negative differential resistance, and other properties of possible importance in molecular scale electronics. In contrast to most reported cases of single-molecule electronics, the electrochemical bridge group tunneling contacts moreover operate in condensed matter environment (as opposed to ultrahigh vacuum) and at room temperature (as opposed to cryogenic temperatures).

The theory of these processes goes back to reports in the 1970s on bridge-assisted electron transfer in the bulk of polar media and at the electrodes. ${ }^{1-9}$ A crucial factor determining the peculiarities of these processes is a strong interaction of the transferable electron with polar environment (phonons) and/or local vibrational modes. This interaction plays a decisive role also in the electron tunneling between two metals

\footnotetext{
a) Author to whom correspondence should be addressed. Electronic mail: theor@elchem.ac.ru
}

through a bridge molecular redox group. The mere existence of two valence states of the redox group at a fixed electric potential (a bistability property) is a result of strong interaction of this group with vibrational subsystem. This was recognized in the first theoretical studies of electron tunneling through a single molecular redox group ${ }^{10-12}$ where one of the electron tunneling mechanisms in such systems was described, viz., stepwise sequential electron transfer (ET) through a relaxed intermediate state when the coupling of the bridge group with both electrodes is weak (further elaborated). ${ }^{13}$ The current was expressed through the rate constants (transition probabilities) for the individual ET steps, and the limit of diabatic ET (employing the Fermi golden rule $)^{14,15}$ and the high-temperature limit were in focus. The strong interaction of the redox group with the vibrational subsystem results in the appearance of the FranckCondon factor in the expressions of the transition probabilities. Essentially the same approach was used much later in theoretical analysis of stepwise sequential electron tunneling through solid state contacts in the low-temperature limit. ${ }^{16,17}$ The notion of Franck-Condon blockade of the tunneling process ${ }^{16,17}$ is thus identical to the appearance of the Franck-Condon factor in the earlier reports. A similar mechanism, viz., inelastic electron tunneling with the excitation of several phonons has been discussed in Refs. 18 and 19 where the expansion in the powers of the coupling constant characterizing the interaction of the electrons with phonons was 
used. This representation is valid in the weak coupling limit in the case when the oxidized state is considered as the only state of the bridge group.

Experimental investigations of single-molecule electrochemical redox switches was initiated by Tao's report. ${ }^{20}$ This work disclosed tunneling current features of a single redox molecule (iron protoporphyrin IX) with a maximum in the current dependence on the overpotential. An attempt of theoretical explanation of these data was offered in Ref. 21 using concepts of resonant or off-resonant electron tunneling averaged over the distribution function of free phonons. Electron tunneling only through a single, i.e., the oxidized state of the redox group was thus considered, with emphasis on the inverted free energy region. However, as shown ${ }^{11,12}$ and in later reports ${ }^{22-29}$ incorporation of both valence states is of principal importance in a full theoretical formalism.

A new physical mechanism of electron tunneling in in situ bridged contacts when the interaction of the redox group with both metals is strong was reported in Ref. 30. A "boost" of electrons, i.e., up to hundred(s) electrons can then be transferred between the metals via the redox molecule in a single in situ STM event, while the bridge group redox level passes through the energy window between the Fermi levels of the metals. An important step in the understanding of the ET mechanism in these systems was offered in Ref. 23 where the crucial role of the redox transformations and vibrational relaxation of the bridge group confined in the tunnel gap was documented and the relationships between the tunneling current and electrode potential fully established.

The spectroscopic current/overpotential relation combined with the expectations of an electronic "boost" was supported by new data and analysis for a group of bipy-based Os complexes (bipy $=2,2^{\prime}$-bipyridine). ${ }^{31,32}$ The importance of the multielectron boost is further strikingly illuminated by the substantial difference between the many-ET process of the Os complex and the single-ET behavior of analogous Co complex. ${ }^{31}$

One of the most interesting theoretical outcomes was the prediction of the maximum of the tunneling current/ overpotential dependence near the equilibrium potential of the redox group at fixed values of the bias voltage, ${ }^{23} \mathrm{cf}$. the experimental data of Tao. ${ }^{20}$ This expectation has been supported by a number of recent experimental studies. ${ }^{31-38}$ Not only the maximum itself has been clearly observed but the dependence of maximum current and the position of the maximum on the bias voltage has also been studied ${ }^{32,33}$ and analyzed. ${ }^{32}$ Closer examination of Tao's data shows that the current maximum for this system is also close to the equilibrium potential. ${ }^{23,39,40}$ As a whole, the multitude of new data therefore accord broadly with the expectations of the theory. However, the application of the theory to new experimental data also shows the need to include other features in a more detailed analysis. In particular, the Debye screening of the electric potential in the tunnel gap needs to be considered in more detail. As shown recently ${ }^{41-43}$ this is likely to disclose a number of new interesting effects. The aim of the present report is to present both general equations for the tunneling current through a redox group and simple approximate expressions useful for experimental data analysis. The report is

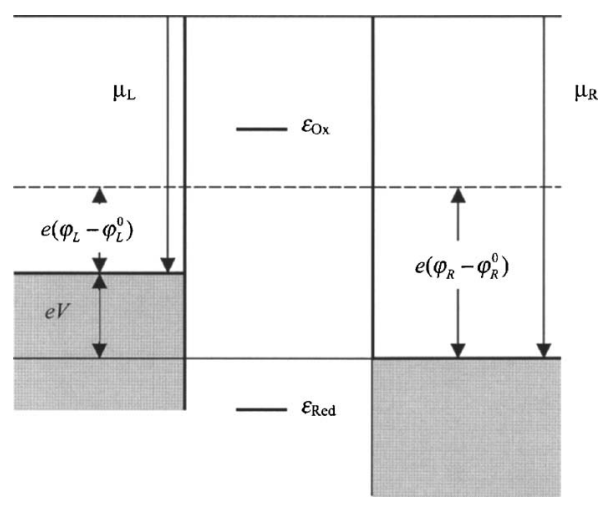

FIG. 1. Energy diagram for the redox mediated tunneling contact. The dashed line shows the position of the electrochemical potentials at equilibrium; equilibrium potentials are defined by Eqs. (8) and (9).

organized as follows. The model and general relationships for nonadiabatic electron transitions are described in Sec. II. The relationship between the reaction free energies of the transition is established in Sec. III. The effects of Debye screening on the potential distribution and the current are discussed in Sec. IV. The limit of strong coupling of the redox group with both electrodes is considered in Sec. V, while Secs. VI and VII offer a discussion of the results and some concluding remarks.

\section{THE MODEL AND THE LIMIT OF WEAK COUPLING OF THE REDOX MOLECULE WITH THE METALS}

The system consists of two metal electrodes (left, $L$, and right, $R$ ) immersed into the electrolyte solution and a molecule with a redox group, B, confined in the gap between the electrodes. Two potential differences can be controlled independently: the bias voltage between the metals $V$ and the potential of the left $\left(\varphi_{L}\right)$ or right $\left(\varphi_{R}\right)$ electrode with respect to the potential in the bulk of the solution $\left(\varphi_{s}\right)$. The latter will be taken to be zero $\left(\varphi_{s}=0\right)$ throughout (Fig. 1). The case where the Fermi level of the left electrode is located higher than that of the right electrode, i.e., positive bias voltage, will be specifically considered. Extension to negative bias voltages is straightforward.

We consider first the case where the interaction of the redox group with both metals is weak, corresponding to nonadiabatic electronic transitions. The electron tunneling mechanism in this case is of a stepwise character. We start from the state where the redox group is, e.g., in the oxidized state. ET from the left metal to the redox group, i.e., reduction of the redox group, is then the first step. The thermal fluctuations of the molecular environment are crucial and take the electronic energy level $\varepsilon_{B}$ towards the Fermi level of the left metal $\varepsilon_{F L}$. Following the first ET step, the molecular level relaxes fully to a new position below the Fermi level of the right metal $\varepsilon_{F R}$.

This configuration would prevail for a long time at low temperatures and could here serve as a memory element. However, at room temperature, due to renewed thermal fluctuations of the molecular environment the occupied electronic energy level will approach resonance with unoccupied 
energy levels of the right metal. ET to these levels will then reoxidize the redox group thus completing the ET cycle from the left to the right metal.

The electric current through the contact following this mechanism can be given as ${ }^{23,27}$

$$
j=e \frac{k_{L B} k_{B R}-k_{B L} k_{R B}}{k_{L B}+k_{B L}+k_{R B}+k_{B R}},
$$

where $k_{L B}$ and $k_{B R}$ are the rate constants for the ET from the left metal to the redox group and from the redox group to the right metal, respectively. $k_{B L}$ and $k_{R B}$ are the rate constants for the reverse transitions.

The simplest expressions for the tunneling current emerge in the so-called spinless model where the electron spin is ignored. The spinless model is used in most theoretical reports on one-electron electrochemical reactions. The implicitly assumed or explicitly claimed single occupancy of the molecular orbitals has been rationalized by infinitely large repulsion energy, $U$, for two electrons with opposite spin directions on a single electronic energy level in the redox molecule. It will be shown below, however, that the assumption of infinitely large repulsion energy is not the same as the spinless model. The double spin degeneracy of the electronic energy levels in the metal electrode means that ET from a given energy level in the metal to the redox level can involve transition of either of two electrons (with oppo- site spins) from the occupied electronic energy level in the metal. At the same time this is not the case for the reverse transition of one electron occupying the redox energy level. This effect will be taken into account below.

According to the detailed balance principle the rate constants of the forward and reverse transitions are related to each other as ${ }^{14,15}$

$$
k_{B L}=\frac{1}{2} k_{L B} \exp \left(-\frac{\Delta F_{B L}}{k_{\mathrm{B}} T}\right), \quad k_{R B}=2 k_{B R} \exp \left(-\frac{\Delta F_{R B}}{k_{\mathrm{B}} T}\right),
$$

where $\Delta F_{B L}$ and $\Delta F_{R B}$ are the reaction free energies of the electronic transition from the redox group to the Fermi level of the left metal $\varepsilon_{F L}$ and from $\varepsilon_{F R}$ to the redox group, respectively, with spin degeneracy disregarded. The appearance of the factors 2 and $\frac{1}{2}$ is due exactly to the difference in the occupation of the electronic energy levels in the metal (two electrons with opposite spins) and in the redox group (one electron only) [see also Eq. (A1)]. The reaction free energies are related to each other as

$$
\Delta F_{B R}=e V-\Delta F_{B L},
$$

with $V$ being the bias voltage, i.e., the difference of electrochemical potentials of the two metals, Fig. 1.

With the use of Eqs. (2) and (3) the current expression can be transformed to

$$
j_{n, \text { ad }}=e \frac{2 k_{L B} k_{B R} \sinh \left(e V / 2 k_{\mathrm{B}} T\right)}{k_{L B}\left[\exp \left(e V / 2 k_{\mathrm{B}} T\right)+\exp \left(\left(e V / 2-\Delta F_{B L}^{(2)}\right) / k_{\mathrm{B}} T\right)\right]+k_{B R}\left[\exp \left(e V / 2 k_{\mathrm{B}} T\right)+\exp \left(-\left(e V / 2-\Delta F_{B L}^{(2)}\right) / k_{\mathrm{B}} T\right)\right]},
$$

where the reaction free energy $\Delta F_{B L}^{(2)}$ is related to $\Delta F_{B L}$ as $\Delta F_{B L}^{(2)}=\Delta F_{B L}+k_{B} T \ln 2$.

The reaction free energy $\Delta F_{B L}$ depends on the electrode potentials

$$
\begin{aligned}
\Delta F_{B L}= & \varepsilon_{F L}-\varepsilon_{B}-\Delta F_{\text {solv }}-e\left[\varphi_{L}-\varphi_{s}\right] \\
& +e\left[\psi\left(z ; \varphi_{L}-\varphi_{L}^{p z c}, \varphi_{R}-\varphi_{R}^{p z c}\right)-\varphi_{s}\right],
\end{aligned}
$$

where the Fermi levels $\varepsilon_{F L}$ and $\varepsilon_{F R}$ are counted from the energies $-e \varphi_{L}$ and $-e \varphi_{R}\left(\varphi_{L}\right.$ and $\varphi_{R}$ are the Galvani potentials of the left and right electrodes, respectively), and $\varepsilon_{B}$ is the electronic energy in the bridge group counted from the energy $-e \varphi_{s}$, where $\varphi_{s}$ is the potential in the bulk of the solution. As noted, below the potential $\varphi_{s}$ will be taken to be zero throughout. $\Delta F_{\text {solv }}$ is the difference of the solvation free energies of the redox group in the reduced and oxidized states and $\psi\left(z ; \varphi_{L}-\varphi_{L}^{p z c}, \varphi_{R}-\varphi_{R}^{p z c}\right)$ the potential at the site of the redox group. This potential depends on the Galvani potentials $\varphi_{L}$ and $\varphi_{R}$ and on the potentials of zero charge $\varphi_{L}^{p z c}$ and $\varphi_{R}^{p z c}$ of the corresponding electrodes.

The rate constants are calculated with the use of the Fermi golden rule and integration over all electronic energy levels in the corresponding metal [Eqs. (A1), (A2), (A6), and
(A7) in the Appendix]. Together with Eq. (4) they determine the dependence of the current on the bias voltage and the electrode potentials. As seen from Eq. (5), the behavior of the current depends on the potential distribution in the tunnel gap. Analysis of the equations shows that the current as a function of the electrode potential at fixed bias voltage $V$ has a maximum close to the equilibrium potential. Approximate expressions describing the current in the neighborhood of the maximum can be derived with the use of approximate expressions for the rate constants which emerge from Eqs. (A1) and (A2) at small absolute values of the reaction free energies of the transitions

$$
\begin{aligned}
& k_{L B} \approx 2 k_{L}^{0} \exp \left(-E_{r L} / 4 k_{\mathrm{B}} T\right) \exp \left(\Delta F_{B L} / 2 k_{B} T\right), \\
& k_{B R} \approx k_{R}^{0} \exp \left(-E_{r R} / 4 k_{\mathrm{B}} T\right) \exp \left[\left(e V-\Delta F_{B L}\right) / 2 k_{\mathrm{B}} T\right],
\end{aligned}
$$

where $E_{r L}$ and $E_{r R}$ are the reorganization energies of the molecular environment and

$$
k_{L}^{0}=\frac{\omega}{2 \pi} 2 \pi \kappa_{L} \rho_{L} k_{\mathrm{B}} T, \quad k_{R}^{0}=\frac{\omega}{2 \pi} 2 \pi \kappa_{R} \rho_{R} k_{\mathrm{B}} T .
$$

$\omega$ is effective frequency of the molecular environment, $\rho_{L}$ and $\rho_{R}$ the densities of the electron states in the metals (with 
the neglect of spin degeneracy), and $\kappa_{L}$ and $\kappa_{R}$ are electron transmission coefficients. The latter correspond to onefold passage through the crossing point of the diabatic free energy surfaces [the factor 2 in Eq. (7) takes into account twofold passage through the crossing point in the nonadiabatic case and the factor $\pi$ appears from the integration over the electronic energy spectrum].

Equations (6) are valid when the absolute values of the reaction free energies are much smaller than the corresponding reorganization energies. The approximate expressions for the electric current obtained from Eqs. (6) and (7) are given in the Appendix [Eqs. (A3)-(A5)]. These equations are still rather complex since the dependence of the reaction free energy of transition $\Delta F_{B L}^{(2)}$ on the electrode potentials in general may be quite complicated. The relation of $\Delta F_{B L}^{(2)}$ to the electric potentials is discussed in Sec. III.

\section{REACTION FREE ENERGY OF THE TRANSITION AND THE ELECTRODE POTENTIALS}

The general relationship between the reaction free energy of the transition and the electrode potential is given by Eq. (5). It is convenient to introduce the "equilibrium" potentials $\varphi_{L}^{0}$ and $\varphi_{R}^{0}$ determined by the condition that the currents through each electrode and the reaction free energies of the transition $\Delta F_{B L}^{(2)}$ and $\Delta F_{R B}^{(2)}$ vanish at zero bias voltage. ${ }^{23}$ This gives

$$
\begin{aligned}
& e \varphi_{L}^{0}-e \psi^{0}\left(z ; \varphi_{L}^{0}-\varphi_{L}^{p z c}, \varphi_{R}^{0}-\varphi_{R}^{p z c}\right)-e \phi_{L}^{0}=0, \\
& e \varphi_{R}^{0}-e \psi^{0}\left(z ; \varphi_{L}^{0}-\varphi_{L}^{p z c}, \varphi_{R}^{0}-\varphi_{R}^{p z c}\right)-e \phi_{R}^{0}=0,
\end{aligned}
$$

where standard redox potentials are equal to

$$
\begin{aligned}
& e \phi_{L}^{0}=\varepsilon_{F L}-\varepsilon_{B}-\Delta F_{\text {solv }}+k_{\mathrm{B}} T \ln 2, \\
& e \phi_{R}=\varepsilon_{F R}-\varepsilon_{B}-\Delta F_{\text {solv }}+k_{B} T \ln 2 .
\end{aligned}
$$

The last terms in the right-hand side of Eq. (10) take into account the spin degeneracy of the Fermi levels in the metals.

Equations (8) and (9) give the following equation for determination of the equilibrium potential $\varphi_{L}^{0}$ :

$$
\varphi_{L}^{0}-\psi^{0}\left(z ; \varphi_{L}^{0}-\varphi_{L}^{p z c}, \phi_{R}^{0}-\phi_{R}^{p z c}+\varphi_{L}^{0}-\phi_{L}^{0}\right)=\phi_{L}^{0} .
$$

As seen from Eq. (11), $\varphi_{L}^{0}$ does not in general coincide with the standard redox potential and differs from the latter by the potential at the site of the redox group $\psi^{0}$. We also introduce the "cathodic" overpotential

$$
\eta=\varphi_{L}^{0}-\varphi_{L}
$$

Positive values of $\eta$ mean that the potential of the left electrode is lower than the corresponding equilibrium potential. Taking into account the relationship between the bias voltage and the electrode potentials ${ }^{41}$ (Fig. 1)

$$
V=\varphi_{R}-\varphi_{R}^{0}-\left(\varphi_{L}-\varphi_{L}^{0}\right),
$$

we obtain for the reaction free energy ${ }^{15}$

$$
\begin{aligned}
\Delta F_{B L}^{(2)}= & e\left\{\eta+\psi\left(z ; \varphi_{L}^{0}-\varphi_{L}^{p z c}-\eta ; \varphi_{R}-\varphi_{x}^{p z c}\right)\right. \\
& \left.-\psi^{0}\left(z ; \psi_{L}^{0}-\varphi_{L}^{p z c} ; \varphi_{R}^{0}-\varphi_{R}^{p z c}\right)\right\} .
\end{aligned}
$$

The quantities $\psi$ and $\psi^{0}$ as well as the equilibrium potentials $\varphi_{L}^{0}$ and $\varphi_{R}^{0}$ should be calculated as the solution of the corresponding electrostatic problem. At small values of $V$ and $\eta$ Eq. (14) can be recast approximately as

$$
\Delta F_{B L}^{(2)}=e(\xi \eta+\gamma V),
$$

where

$$
\gamma(z)=\left.\frac{\partial \psi}{\partial \varphi_{R}}\right|_{\varphi_{R}=\varphi_{R}^{0}}, \quad \xi(z)=1-\left.\frac{\partial \psi}{\partial \varphi_{L}}\right|_{\varphi_{L}=\varphi_{L}^{0}}-\gamma(z) .
$$

Equations (15) and (16) are exact only in the case of linear response of the double layer to the electrode potentials.

\section{DEBYE SCREENING EFFECTS ON THE POTENTIAL DISTRIBUTION IN THE TUNNELING GAP AND ON THE TUNNELING CURRENT}

We consider a narrow tunneling gap with a small redox group the effect of which itself on the potential distribution may be neglected. The Poisson-Boltzmann equation is nonlinear when the potential exceeds $k_{\mathrm{B}} T / e$. A number of other effects such as dielectric saturation and spatial dispersion of the dielectric properties of the solvent, the energy of ion transfer from the bulk of the solution, "lattice saturation" effects, etc., could also be important. ${ }^{44}$ However as shown, ${ }^{44}$ most of these effects compensate almost completely each other in a narrow gap. The potential distribution is therefore very close to that obtained as the solution of linearized Poisson-Boltzmann equation. The solution has the following form for a system with planar geometry: ${ }^{44}$

$$
\begin{aligned}
\psi\left(z ; \varphi_{L} ; \varphi_{R}\right)= & \gamma\left(L-z ; L_{D}\right)\left(\varphi_{L}-\varphi_{L}^{p z c}\right) \\
& +\gamma\left(z ; L_{D}\right)\left(\varphi_{R}-\varphi_{R}^{p z c}\right),
\end{aligned}
$$

where $L$ is the width of the tunneling gap, $L_{D}$ the Debye length, and

$$
\gamma\left(z ; L_{D}\right)=\frac{\exp \left(z / L_{D}\right)-\exp \left(-z / L_{D}\right)}{\exp \left(L / L_{D}\right)-\exp \left(-L / L_{D}\right)} .
$$

We obtain for $\xi$ and $\gamma$ in Eq. (16)

$$
\xi(z)=1-\gamma\left(L-z, L_{D}\right)-\gamma\left(z, L_{D}\right), \quad \gamma(z)=\gamma\left(z, L_{D}\right) .
$$

In this case the relationship between $\varphi_{L}^{0}$ and standard potentials $\phi_{L}^{0}$ and $\phi_{R}^{0}$ can also be derived [see Eq. (A14)].

Equations (15), (19), and (A3) give a simple approximate current expression

$$
\begin{aligned}
j_{n, \text { ad }}= & j_{0} \sinh \frac{e V}{2 k_{\mathrm{B}} T} \\
& \times\left\{\exp \left(\frac{e V}{4 k_{\mathrm{B}} T}\right) \cosh \left[\frac{e(0.5-\gamma) V-e \xi \eta}{2 k_{\mathrm{B}} T}-a\right]\right. \\
& \left.+\exp \left(-\frac{e V}{4 k_{\mathrm{B}} T}\right) \cosh \left[\frac{e(0.5-\gamma) V-e \xi \eta}{2 k_{\mathrm{B}} T}+a\right]\right\}^{-1},
\end{aligned}
$$


where $a$ and $j_{0}$ are given by Eq. (A4). The factor $a$ describes possible asymmetry of the contact due to nonsymmetric location of the redox group and different electronic interactions of the redox group with the electrodes. This equation is formally exact if Eqs. (A12) and (A13) are used for $j_{0}$ and $a$.

The current as a function of overpotential $\eta$ has a maximum at $\eta=\eta_{\max } . \eta_{\max }$ depends on the bias voltage and tends to $\eta_{\max }=0$ when $V \rightarrow 0$. The dependence of $\eta_{\max }$ on $V$ can be recast in parametric form as follows:

$$
\begin{aligned}
& \frac{e \xi \eta_{\max }}{2 k_{\mathrm{B}} T}=\left(\frac{1}{2}-\gamma\right) \ln \frac{\sinh (a+u)}{\sinh (a-u)}-u, \\
& \frac{e V}{2 k_{\mathrm{B}} T}=\ln \frac{\sinh (a+u)}{\sinh (a-u)}, \quad a \neq 0,
\end{aligned}
$$

where the running variable $u$ varies within the interval $|u|$ $\leqslant|a|$. The dependence of $\eta_{\max }$ on $V$ can be found in explicit form for large $|V|\left(E_{r}>|V| \gg k T\right)$

$$
\frac{e \xi \eta_{\max }}{2 k_{\mathrm{B}} T}=\left(\frac{1}{2}-\gamma\right) \frac{e V}{2 k_{\mathrm{B}} T} \mp a,
$$

where + and - correspond to negative and positive values of $V$, respectively. Equation (22) holds also in the case when $a=0$.

According to Eq. (22) the slope of the line $\eta_{\max }(V)$ is

$$
S\left(z, L_{D}\right)=\frac{1-2 \gamma\left(z, L_{D}\right)}{2\left[1-\gamma\left(L-z, L_{D}\right)-\gamma\left(z, L_{D}\right)\right]} .
$$

If the redox group is located symmetrically in the tunneling gap $(z=1 / 2)$, then $\gamma\left(z, L_{D}\right)=\gamma\left(L-z, L_{D}\right)$. The slope is therefore equal to $S=1 / 2$ and independent of the Debye length. The quantity $|a|$ in this case is small or zero.

The width of the maximum (at half height) is

$$
\frac{\Delta_{\eta}}{k_{\mathrm{B}} T}=\frac{4 \operatorname{arccosh} 2}{1-\gamma\left(L-z, L_{D}\right)-\gamma\left(z, L_{D}\right)} \text {. }
$$

Unlike the slope, $\Delta_{\eta}$ depends on the Debye length even for a symmetric contact. It should be noted, however, that $\Delta_{\eta}$ is independent of $E_{r}$ and $V$ in the weak coupling limit.

\section{THE STRONG COUPLING LIMIT AND COHERENT MECHANISM OF THE IN SITU STM TRANSITION}

When the interaction of the redox group with both metals is strong the mechanism of the electronic transition changes its character entirely. A boost of a large number of electrons $n$ now tunnel through the energy level of the redox group as it relaxes through the energy window between the Fermi levels of two metals at each reaction step (reduction and oxidation), cf. above and Refs. 30-32. The exact expression for the current can be obtained by averaging the partial tunneling current (calculated at a given position of electronic energy of the redox group) over the thermal distribution of the vibrational coordinates in the adiabatic double-well potential [Eq. (B4)]. ${ }^{40}$ We present approximate equations similar to those in the foregoing section. The reverse transitions may be neglected in the adiabatic limit when $e V \gg k_{\mathrm{B}} T$ and the current is ${ }^{23}$

$$
j=2 e n \frac{k_{L B} k_{B R}}{k_{L B}+k_{B L}},
$$

where

$$
n \approx e V\left(\frac{1}{2 \kappa_{L} \rho_{L}}+\frac{1}{\kappa_{R} \rho_{R}}\right)^{-1} .
$$

We note that the degeneracy of the energy levels in the metal affects the number of electrons transferred in each reduction or oxidation step in this limit. The origin of the factor 2 in the first term in the parentheses is the fact that the energy interval for the transition of one electron from the left metal to the redox group, $\Delta \varepsilon_{L B} \sim 1 /\left(2 \kappa_{L} \rho_{L}\right)$, is one-half of the corresponding energy interval calculated with the neglect of double occupation of a given electronic state in the metal by electrons with opposite spin. In the case of negative bias voltage (opposite direction of the current) the factor 2 is moved from the first to the second term in the parentheses.

An expression for the current similar to Eq. (20) can be derived using Eq. (25) at small overpotential and bias voltage. The expression differs from Eq. (20) only by the form of the constants $j_{0}$ and $a$ now described by Eqs. (B10) and (B11). A quite simple current expression is obtained, if a weak dependence of the reorganization energy on the position of the redox group within the tunnel gap may be neglected ${ }^{22}$

$$
\begin{aligned}
j_{\mathrm{ad}} \approx & e n \frac{\omega}{2 \pi} \exp \left(-E_{r} / 4 k_{\mathrm{B}} T\right) \\
& \times \frac{\exp \left(e V / 4 k_{\mathrm{B}} T\right)}{\cosh \left((e(0.5-\gamma) V-e \xi \eta) / 2 k_{\mathrm{B}} T\right)} .
\end{aligned}
$$

The current/overpotential dependence shows a maximum which is also described by Eq. (22) at $a=0$. It can be shown with the use of Eq. (B4) that the position of the maximum obeys Eq. (22) at arbitrary values of the bias voltage and $\Delta .{ }^{40}$ Equation (27) shows that the slope of the dependence of $\eta_{\max }$ on $V$ in this approximation is also described by Eq. (23). Analysis of Eq. (B4) shows that the same equation is valid at $E_{r}<0.5 \mathrm{eV}, \Delta \ll e V, e V, e \eta \ll E_{r}$.

It should be noted that the effect of spin degeneracy is not in general reduced to a change of the number of electrons transferred, $n$. Equation (27) is valid for small values of the overpotential, bias voltage, and redox group energy level broadening, $\Delta$ (as compared with the reorganization energy). If these values are close to each other, the activation free energy becomes different (as studied in Ref. 40 for the spinless model). The existence of the electron spin and the repulsion energy will cause additional effects to be studied elsewhere.

\section{DISCUSSION}

General equations for the tunneling current given in the Appendix allow calculating the current both in the nonadiabatic and adiabatic limits in the whole range of the bias voltage and overpotential (Figs. 2 and 3). Figure 2 shows the dependence of the current [normalized to $j_{\max }$ $=e k^{0}\left(\pi E_{r} / 2 k_{B} T\right)^{1 / 2}$ at large bias voltage $]$ on the bias voltage 


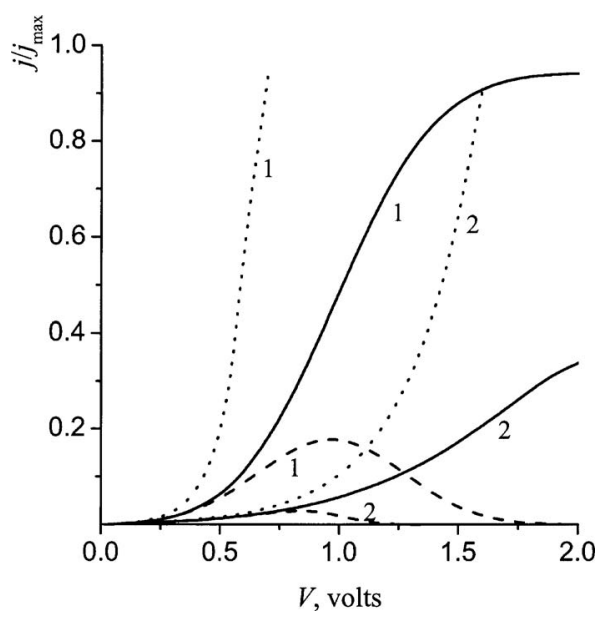

FIG. 2. Dependence of the tunneling current on the bias voltage at $\eta=0$ : (1) $L_{D} / L=1.5$; (2) $L_{D} / L=0.3$. The solid curves are calculated according to the exact equations (1), (2), (4), (A1), (A2), (A12), and (A13); the dotted curves are calculated with the use of Eqs. (20) and (A4); and the dashed curves are calculated according to Eqs. (1), (2), (A8), and (A9). $z / L=0.5$.

for a nonadiabatic process calculated with the use of Eqs. (1), (2), (4), (A1), (A2), (A12), and (A13) and of the approximate Eqs. (20) and (A4). The correlations calculated with the use of the simple quadratic ("Marcus-type") expression for the rate constants, Eqs. (A8) and (A9), are also given. The comparison shows that the simple approximate equation (dotted curves) works well for $e V \ll E_{r}$ as expected. The dependences based on the quadratic rate constant expressions (dashed curves) extend to a slightly wider region. However, they also deviate strongly from the exact solution (solid curves) at large bias voltage, showing a maximum and subsequent fast decay. The exact current/voltage curve reaches a plateau value rather rapidly at large $L_{D} / L$ whereas the current dependence on the bias voltage is much weaker at small $L_{D} / L$ values. The latter is due to strong screening of the potential by the electrolyte, resulting in small values of $\gamma$ (e.g., $\gamma$ $=0.47$ and 0.2 for $L_{D} / L=1.5$ and 0.3 , respectively) and therefore, in a weak dependence of the position of the electronic energy level of the redox group on $V$. Figure 3 shows the dependence of the current (normalized to $k^{0} \sqrt{4 \pi E_{r}}$ where $k^{0}=k_{L}^{0}=k_{R}^{0}$ and $E_{r}=E_{r L}=E_{r R}$ ) on the overpotential calculated with the use of the exact equations. It is seen that current

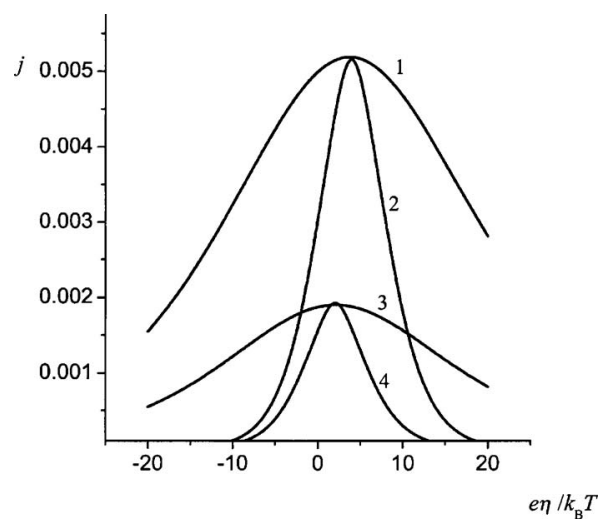

FIG. 3. Dependence of the tunneling current on the overpotential. The current is normalized to $k^{0} \sqrt{4 \pi E_{r}}$ (see text), $E_{r}=0.5 \mathrm{eV} ; z / L=0.5$. (1) $\mathrm{eV}$ $=0.2 \mathrm{eV} ; L_{D} / L=0.8$; (2) $0.2 \mathrm{eV} ; 0.3$; (3) $0.1 \mathrm{eV} ; 0.8$; (4) $0.1 \mathrm{eV} ; 0.3$.

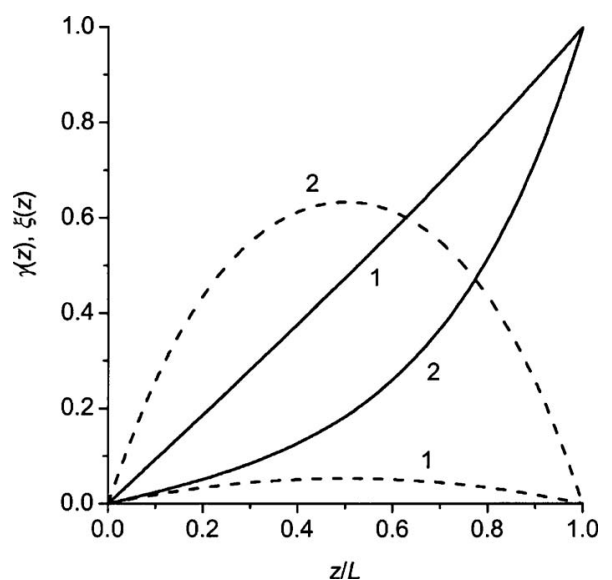

FIG. 4. Dependence of the coefficients $\gamma(z)$ (solid lines) and $\xi(z)$ (dashed lines) on the position of the redox group in the tunneling gap at different values of the Debye length $L_{D}$. (1) $L_{D} / L=1.5$, (2) $L_{D} / L=0.3$.

passes a maximum near the equilibrium potential. The maximum is narrower when the screening is stronger. A more detailed analysis of the current behavior in the neighborhood of the maximum can be performed with the use of the approximate equations obtained within linear electrostatics [Eqs. (20)-(24) and (27)]. These involve the overpotential and bias voltage in the combination $\eta_{v}=\xi \eta+(\gamma-0.5) V$, where the quantities $\xi$ and $\gamma$ depend both on the position of the redox group $z$ and on the Debye length $L_{D}$ [Eq. (19)]. Typical $\xi(z)$ and $\gamma(z)$ curves are shown in Fig. 4. The quantity $\gamma$ quantifies the effect of variation of the potential of the right electrode on the potential at the site of the bridge group and therefore increases monotonously with increasing $z$. The quantity $\xi$ quantifies the effect of variation of the potentials of both electrodes and therefore passes through a maximum. Typical dependences of the position of the maximum of the tunneling current on the bias voltage are shown in Fig. 5. All curves cross at the point $\eta_{\max }=0, V=0$. The slope is well described by the approximate Eq. (23) at small $a$ values whereas it reaches a limiting value at larger bias voltage when $a$ is large.

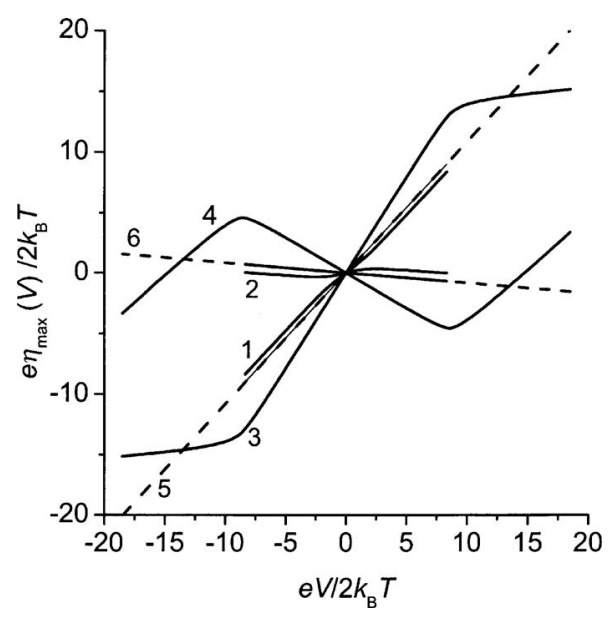

FIG. 5. Dependence of the position of the maximum of the current/ overpotential relation on the bias voltage at different positions of the redox group $z$ and the parameter $a$ calculated according to Eq. (21) (solid lines). The dashed lines are calculated according to Eq. (22) at corresponding positions of the redox group and $a=0 . L_{D} / L=0.5$. (1) $z / L=0.3, a=0.2 ;$ (2) $z / L=0.7, a=-0.2$; (3) $z / L=0.3, a=5$; (4) $z / L=0.7, a=-5$. 


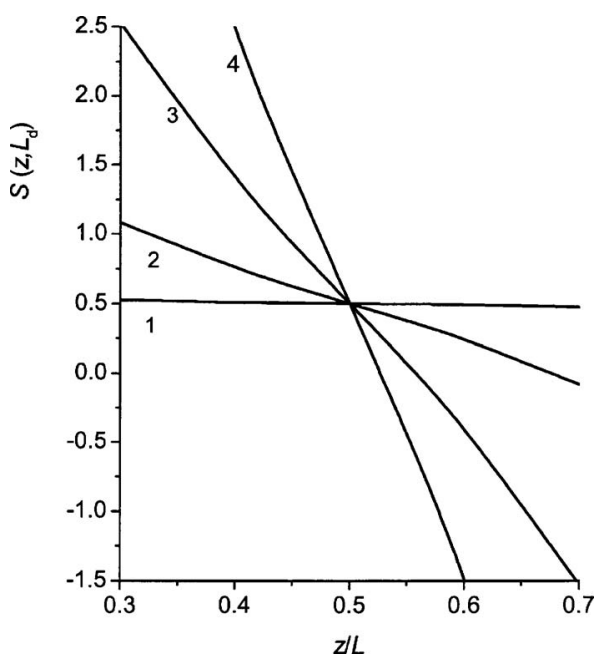

FIG. 6. Dependence of the slope of the line $\eta_{\max }(V)$ on the position of the redox group at different values of the Debye length. $L_{D} / L$ $=0.1(1) ; 0.5(2) ; 1(3) ; 1.5(4)$.

The dependence of the slope on the position of the redox group is shown in Fig. 6. The slope is close to $\frac{1}{2}$ for small values of the Debye length (strong screening) and practically independent of the position of the redox group within the interval between $0.3 \mathrm{~L}$ and $0.7 \mathrm{~L}$. The dependence is stronger at larger values of the Debye length. The slope is also larger if the redox group is located closer to the left electrode. This asymmetry is caused by the choice of the left electrode as the substrate (with corresponding consequences for the potential variations).

The slope depends on two quantities, $\gamma\left(z ; L_{D}\right)$ and $\gamma\left(L-z ; L_{D}\right)$, Eq. (23). In order to determine these separately from experimental data we need an additional relationship, determined by the width of the maximum $\Delta_{\eta}$, Eq. (24) (Fig. 7). As seen from Fig. 7, the width increases with increasing Debye length. Within the approximations used the width is independent of the bias voltage and determined by the same quantities as the slope. The use of experimental data for the slope and the width of the maximum therefore allows determining $\gamma\left(z ; L_{D}\right)$ and $\gamma\left(L-z ; L_{D}\right)$

$$
\begin{aligned}
& \gamma\left(z, L_{D}\right)=\frac{1}{2}-\frac{4 S}{\Delta_{\eta}} \operatorname{arccosh} 2, \\
& \gamma\left(L-z, L_{D}\right)=1-\frac{1}{2 S}-\left(1-\frac{1}{S}\right) \gamma\left(z, L_{D}\right) .
\end{aligned}
$$

The use of the dependences of $\gamma\left(z ; L_{D}\right)$ and $\gamma\left(L-z ; L_{D}\right)$ on $z$ and $L_{D}$ [Eqs. (18) and (19)] allows in turn estimating the position of the redox group in the tunneling gap.

The current expressions in the nonadiabatic limit take into account only sequential electronic transitions with the probabilities calculated in first order in the interactions with the metals. The adiabatic tunneling current involves both resonance and off-resonance electron tunneling. The latter is commonly denoted as superexchange. This notion goes back to the early theory of bridge-assisted ET in bulk solution. ${ }^{9}$ Similar processes in solid state contacts have been denoted "cotunneling." 45

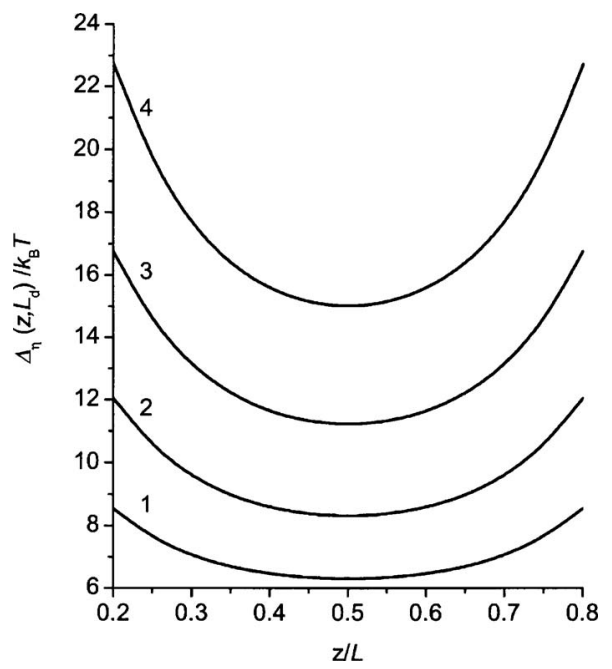

FIG. 7. Dependence of the width of the current/overpotential maximum on the position of the redox group at different values of the Debye length. $L_{D} / L=0.2(1) ; 0.3(2) ; 0.4(3) ; 0.5(4)$.

As noted in Sec. I, reports of experimental work on in situ tunneling through a redox molecule have appeared recently $^{32,38}$ showing a strong tunneling current maximum close to the equilibrium potential. The dependence of the position of the maximum on the bias voltage was studied in Refs. 31 and 33. The dependence of the maximum tunneling current position on the bias voltage was fairly linear with the slope close to -0.5 for the Osmium complexes. ${ }^{31}$ Taking into account that the data were plotted with the use of the ordinary overpotential $\eta_{a}=\varphi_{L}-\varphi_{L}^{0}$, the sign of the slope must be changed when Eq. (23) is used. As noted, the slope 0.5 is typical for a symmetric contact where $\gamma\left(z ; L_{D}\right)=\gamma\left(L-z ; L_{D}\right)$ $=\gamma\left(L / 2 ; L_{D}\right)$. Unfortunately there were not enough data to determine the absolute value of $\gamma\left(L / 2 ; L_{D}\right)$. The Os-based system also showed the "boost" of a large number of electrons expected when the electronic interaction between the redox molecule and the enclosing electrodes is strong. ${ }^{32}$

The tunneling current through a single azurin molecule attached to a gold electrode also showed an approximately linear dependence of the maximum position on the bias voltage with the slope approximately equal to $-0.5 .^{33}$ This would correspond to a redox center located symmetrically within the tunneling gap ( $S=1 / 2$ in the terms of the present report) or to another location in the case of strong screening $(S \approx 1 / 2)$. In spite of the scatter of the data one may make some observations concerning the width and the height of the maximum. The width of the maximum increases with the increase of the bias voltage (from 50 to $300 \mathrm{mV}$ ). The height of the maximum also increases which is in line with general predictions of the theory. These results were fitted in Ref. 33 the use of the approximate equations (20) (with $a=0$ ) within the sequential stepwise mechanism. The electronic coupling matrix element was also used as a fitting parameter. However, there are no physical reasons to assume that the matrix element depends significantly on the bias voltage.

Instead we calculated current/overpotential dependences at different values of bias voltage with the use of exact equations for the strong and weak coupling limits [Eqs. (1), (A1), (A2), and (B4)-(B8)] keeping the electronic matrix element 


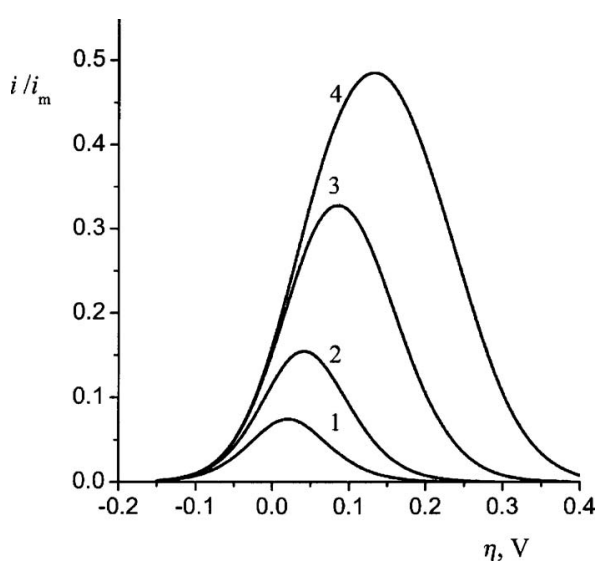

FIG. 8. The current/overpotential dependence in the totally nonadiabatic regime. Calculated according to Eqs. (1), (A1), and (A2) with $E_{r}=0.1 \mathrm{eV}$ for different values of the bias voltage, $i_{m}=k_{L}^{0} ; k_{L}^{0}=k_{R}^{0}$. (1) $V=50 \mathrm{mV}$; (2) $V$ $=100 \mathrm{mV}$; (3) $V=200 \mathrm{mV}$; (4) $V=300 \mathrm{mV}$.

(and therefore $\Delta$ ) constant. Figures 8 and 9 show that the experimental data of Ref. 33 are reproduced rather well with respect to the width of the current/overpotential maximum. It was assumed that the Debye screening is strong so that $\gamma$ $\approx 0$ and $\xi \approx 1$. The reorganization energy was chosen to be $0.1 \mathrm{eV}$ in both limits. However, the ratios of the current maximum values for the values of the bias voltage 300 and $50 \mathrm{mV}$ are about twice as large as the experimental value in both cases. Thus these calculations do not allow discriminating between the weak and strong coupling limits for the experimental data of Ref. 33 .

\section{CONCLUDING REMARKS}

The present work follows previous theoretical reports on tunneling spectroscopy of redox molecules in solution when the molecule is confined either in an in situ STM tunneling gap or in the gap between a pair of nanoelectrodes, but extends this work in important respects. The formalism is first generalized relative to the previous formalism particularly in the sense that the tunneling current forms apply to the whole range of overpotential and bias voltage, in either the nonadiabatic or adiabatic limits. There is, for example, no need

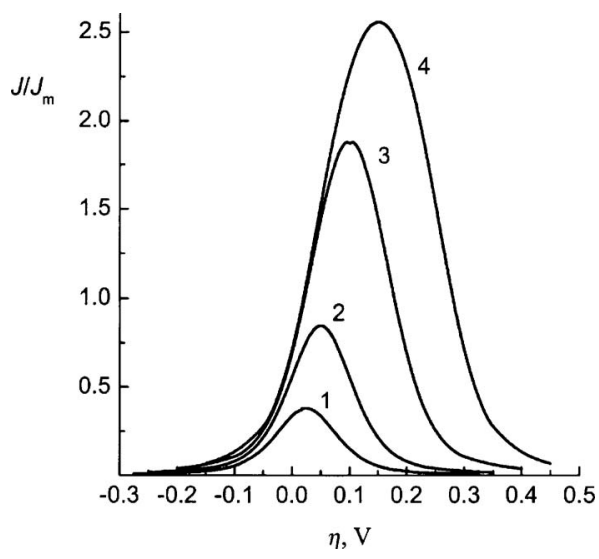

FIG. 9. The current/overpotential dependence in the totally adiabatic regime. Calculated according to Eqs. (B4)-(B8) with $E_{r}=0.1 \mathrm{eV}$ and $\Delta$ $=0.025 \mathrm{eV}$ for different values of the bias voltage, $j_{m}=2 e \Delta_{L} \Delta_{R} / \pi \hbar \Delta, \Delta_{L}$ $=\Delta_{R}$. for resorting to interpolation formulas ${ }^{29}$ between the limits of small and large bias voltages (although the analysis shows that the interpolation formula qualitatively rather well describes the current in the neighborhood of the maximum, differing from the exact one by a factor of 2-3). The generalized form is represented by Eq. (1) or the simpler Eq. (25) (with $n=\frac{1}{2}$ ), combined with Eqs. (A1) and (A2) for the nonadiabatic limit, with concomitant single-electron transfer in the individual in situ STM event. The general nonadiabatic tunneling current form can also be given the form in Eq. (A3) combined with Eqs. (A12) and (A13). The corresponding general tunneling current forms in the adiabatic limit are given by Eqs. (B4)-(B9).

The general formalism reduces, secondly to attractive simplified forms subject to constraints which can, however, be expected to accord with experimentally accessible conditions. Such constraints are, for example, small driving forces compared with the reorganization free energy, reducing the general rate constant forms to Eqs. (4)-(7) combined with Eqs. (A3) and (A4) in the nonadiabatic limit and Eq. (27) in the adiabatic limit. Merits of these limiting equations are their immediate suitability for experimental data analysis. Simple approximate equations allow one to calculate the tunnel current in the neighborhood of its maximum whereas the exact ones given in the Appendix describe the current in the whole range of the variation of the bias voltage and overpotential.

A third outcome of the study is the incorporation of ionic screening effects in the description of the potential distribution in the tunneling gap. The Debye (Gouy) approximation was used. Nonlinearity of the Poisson-Boltzmann equation and ionic finite-size effects were shown in previous studies to largely cancel each other ${ }^{41-44}$ but these studies addressed superexchange of nonredox molecular tunneling processes and did not extend to redox molecules. The potential distribution is given by Eqs. (17)-(19) which can be combined directly with the general tunneling current forms in the nonadiabatic [Eqs. (4)-(7), (20), and (A3)-(A5)] and adiabatic limits [Eq. (27)]. A potentially very useful basis for ionic strength analysis is, however, achieved by the introduction of the bias voltage dependence of maximum position, $\eta_{\max }$, and the maximum width, $\Delta_{\eta}$, in the tunneling current/ overpotential correlation at different ionic strengths. These quantities are represented approximately by Eqs. (21) and (24), to which adds the slope of the $\eta_{\max }(V)$ correlation in Eq. (23). These equations are only limited by the constraint of dielectric linearity of the solvent in the electrochemical double layers of the two electrodes.

The dependence $\eta_{\max }(V)$ may in general be nonmonotonous but is linear for large bias voltages with a slope described by Eq. (23). Equations (23) and (24) show that the limiting value of the slope of the function $\eta_{\max }(V)$ and the width $\Delta_{\eta}$ depend on the position of the redox group $z$ and on the screening of the electric potential in the tunneling gap. At small and moderate screening the slope increases with a shift of the redox group towards the left electrode (substrate). The slope is close to $\frac{1}{2}$ at strong screening and depends here rather weakly on the position of the redox group.

Although the approximate expressions for the current 
and the conclusions regarding the slope and width of the function $\eta_{\max }(V)$ were derived in the approximation of linear electrostatics, the main qualitative trends remain also for nonlinear screening. For example, as follows from Eq. (16), the stronger the screening, the smaller is $\gamma(z)$ whereas $\xi(z)$ gets close to unity. The slope $S$ [Eq. (23)] is therefore equal to 0.5 for strong nonlinear screening and is independent of the position of the redox group. In the case of nonlinear screening, the quantities $\gamma(z)$ and $\xi(z)$ may also depend on the electrode potentials (in particular, on the bias voltage). This may result in an additional dependence of the width of the maximum on the electrode potentials at large potentials.

The effect of spin degeneracy on the electronic energy levels in the metals is rather small and may be neglected in the first approximation for nonadiabatic transitions. The effect can be much more significant for adiabatic ET due to the change of the height of the activation barrier on the adiabatic free energy surface, again caused by the large value of the repulsion energy $U$ such as shown for ordinary ET reactions involving only a single metal/solution interface. ${ }^{46}$

Electrochemically based tunneling current/overpotential and current/bias voltage relations for confined redox molecules involve, finally, invariably inert ions, sometimes in substantial concentrations. A need for large bias voltages, exceeding or comparable to the reorganization free energy can also be expected frequently. The formalism offers, concluding, a useful comprehensive frame, both for precise data analysis of broader ranges particularly of the bias voltage, without the need of introducing interpolation formulas such as shown previously. ${ }^{29,47}$ In these respects the formalism also offers a facile route to systematic ionic strength analysis or assessment of ionic strength effects via the $\eta_{\max }(V)$ correlation, and explicitly the width and slope of this correlation.

\section{ACKNOWLEDGMENTS}

The work was partially supported by Russian Foundation for Basic Research (Grant No. 06-03-32193). One of the authors (J.U.) acknowledges financial support from the Danish Research Council for Technology and Production Sciences (Contract No. 26.00.0034).

\section{APPENDIX A: GENERAL RELATIONSHIPS FOR NONADIABATIC TRANSITIONS}

General expressions for the electrochemical rate constants of nonadiabatic ET reactions have the form ${ }^{14,15}$

$$
\begin{aligned}
k_{L B}= & 2 k_{L}^{0} \int \frac{d \varepsilon}{k_{\mathrm{B}} T} f_{L}(\varepsilon) \\
& \times \exp \left\{-\left[E_{r L}-\Delta F_{B L}-\left(\varepsilon-\varepsilon_{F L}\right)\right]^{2} / 4 E_{r B} k_{\mathrm{B}} T\right\}, \quad(\mathrm{A} 1) \\
k_{B R}= & k_{R}^{0} \int \frac{d \varepsilon}{k_{\mathrm{B}} T}\left[1-f_{R}(\varepsilon)\right] \\
& \times \exp \left\{-\left[E_{r R}-e V+\Delta F_{B L}+\left(\varepsilon-\varepsilon_{F R}\right)\right]^{2} / 4 E_{r R} k_{\mathrm{B}} T\right\},
\end{aligned}
$$

where $f_{L}(\varepsilon)$ and $f_{R}(\varepsilon)$ are the Fermi distribution functions of the left and right metals, respectively. The origin of the factor 2 in Eq. (A1) is the same as in Eq. (2).

The approximate equations for the rate constants (6) and (7) are obtained from Eqs. (A1) and (A2) if $\left|\Delta F_{B L}\right| \ll E_{r L}$ and $\left|e V-\Delta F_{B L}\right| \ll E_{r R}$. The current expression is then reduced to

$$
j_{n, \mathrm{ad}}=j_{0} \frac{\sinh \left(e V / 2 k_{\mathrm{B}} T\right)}{\exp \left(e V / 4 k_{\mathrm{B}} T\right) \cosh \left[\left(e V / 2-\Delta F_{B L}^{(2)}\right) / 2 k_{\mathrm{B}} T-a\right]+\exp \left(-e V / 4 k_{\mathrm{B}} T\right) \cosh \left[\left(e V / 2-\Delta F_{B L}^{(2)}\right) / 2 k_{\mathrm{B}} T+a\right]},
$$

where

$$
\begin{aligned}
& j_{0}=e \frac{\omega}{2 \pi} 2 \pi \sqrt{2 \kappa_{L} \rho_{L} \kappa_{R} \rho_{R}} k_{\mathrm{B}} T \exp \left(-\bar{E}_{r} / 4 k_{\mathrm{B}} T\right), \\
& a=\ln \left\{\sqrt{\frac{\kappa_{L} \rho_{L}}{\kappa_{R} \rho_{R}}} \exp \left[\frac{E_{r R}-E_{r L}}{8 k_{\mathrm{B}} T}\right]\right\}, \quad \bar{E}_{r}=\frac{1}{2}\left(E_{r L}+E_{r R}\right) .
\end{aligned}
$$

We have, for a symmetric contact $\left(\kappa_{L}=\kappa_{R}=\kappa ; E_{r R}=E_{r L}\right.$ $\left.=E_{r} ; \rho_{L}=\rho_{R}=\rho\right) a=0$,

$$
\begin{aligned}
j_{n, \mathrm{ad}}= & e \frac{\omega}{2 \pi} 2 \sqrt{2} \pi \kappa \rho k_{\mathrm{B}} T \\
& \times \exp \left(-E_{r} / 4 k_{\mathrm{B}} T\right) \frac{\sinh \left(e V / 4 k_{\mathrm{B}} T\right)}{\cosh \left(e V / 2-\Delta F_{B L}^{(2)}\right) / 2 k_{\mathrm{B}} T} .
\end{aligned}
$$

In the general case Eqs. (A1) and (A2) can be rewritten in the form

$$
\begin{aligned}
k_{L B}= & 2 k_{L}^{0} \exp \left\{-\left[E_{r L}-\Delta F_{B L}\right]^{2} / 4 E_{r L} k_{\mathrm{B}} T\right\} \\
& \times \int \frac{d x \exp \left[x\left(1-\Delta F_{B L} / E_{r L}-k_{\mathrm{B}} T x / E_{r L}\right)\right]}{1+\exp (2 x)}, \\
k_{B R}= & k_{R}^{0} \exp \left\{-\left[E_{r R}-e V+\Delta F_{B L}\right]^{2} / 4 E_{r R} k_{\mathrm{B}} T\right\} \\
& \times \int \frac{d x \exp \left\{x\left[1-\left(\Delta F_{B L}-e V\right) / E_{r R}-k_{\mathrm{B}} T x / E_{r R}\right]\right\}}{1+\exp (2 x)} .
\end{aligned}
$$

The exponential factors in front of the integrals in Eqs. (A6) and (A7) represent the usual quadratic free energy forms. If the values of the reorganization energies in the integral terms are increased to infinity, Eqs. (A6) and (A7) take the well known quadratic form 


$$
\begin{aligned}
& k_{L B}=\pi k_{L}^{0} \exp \left\{-\left[E_{r L}-\Delta F_{B L}\right]^{2} / 4 E_{r L} k_{\mathrm{B}} T\right\}, \\
& k_{B R}=(\pi / 2) k_{R}^{0} \exp \left\{-\left[E_{r R}-e V+\Delta F_{B L}\right]^{2} / 4 E_{r R} k_{\mathrm{B}} T\right\} .
\end{aligned}
$$

These equations involve the inverted region at large overpotentials and bias voltages. However, the correct expressions have no inverted region because the integral factors suppress the increase of the activation free energies. These integrals $\left(I_{L}\right.$ and $\left.I_{R}\right)$ thus behave as

$$
\begin{aligned}
& I_{L} \approx\left(\frac{\pi E_{r L}}{k_{B} T}\right)^{1 / 2} \exp \left\{\left[E_{r L}-\Delta F_{B L}\right]^{2} / 4 E_{r L} k_{\mathrm{B}} T\right\}, \\
& I_{R} \approx\left(\frac{\pi E_{r R}}{k_{B} T}\right)^{1 / 2} \exp \left\{\left[E_{r R}-e V+\Delta F_{B L}\right]^{2} / 4 E_{r R} k_{\mathrm{B}} T\right\},
\end{aligned}
$$

in the limit when $\left|\Delta F_{B L}\right| \gg E_{r L}$ and $\left|e V-\Delta F_{B L}\right| \gg E_{r R}$. The expression for the tunneling current in the general case is formally identical to Eq. (A3) with different values for the parameters $j_{0}$ and $a$,

$$
\begin{aligned}
j_{0}= & e \frac{\omega}{2 \pi} 2 \pi \sqrt{2 \kappa_{L} \rho_{L} \kappa_{R} \rho_{R} I_{L} I_{R}} k_{\mathrm{B}} T \exp \left(-\bar{E}_{r} / 4 k_{\mathrm{B}} T\right) \\
& \times \exp \left\{-\left(\Delta F_{L B}^{(2)}-k_{\mathrm{B}} T \ln 2\right)^{2} /\left(8 k_{\mathrm{B}} T E_{r L}\right)\right. \\
& \left.\left.-\left(e V-\Delta F_{L B}^{(2)}+k_{\mathrm{B}} T \ln 2\right)^{2}\right] /\left(8 k_{\mathrm{B}} T E_{r R}\right)\right\}, \\
a= & \ln \left\{\sqrt{\frac{\kappa_{L} \rho_{L} I_{L}}{\kappa_{R} \rho_{R} I_{R}}} \exp \left[\frac{E_{r R}-E_{r L}}{8 k_{\mathrm{B}} T}\right]\right\} \\
& -\frac{\left(\Delta F_{L B}^{(2)}-k_{\mathrm{B}} T \ln 2\right)^{2}}{8 E_{r L} k_{\mathrm{B}} T}+\frac{\left(e V-\Delta F_{L B}^{(2)}+k_{\mathrm{B}} T \ln 2\right)^{2}}{8 E_{r R} k_{\mathrm{B}} T} .
\end{aligned}
$$

The parameter $a$ is a function of the bias voltage in the general case.

The relationship between the equilibrium potentials in the case of linear electrostatics is of the form
$\varphi_{L}^{0}=\left\{[1-\gamma(z)] \phi_{L}^{0}+\gamma(z) \phi_{R}^{0}-\gamma(L-z) \varphi_{L}^{p z c}-\gamma(z) \varphi_{R}^{p z e}\right\} / \xi(z)$.

\section{APPENDIX B: ADIABATIC TRANSITIONS}

When the interaction of the redox group with both electrodes is strong, the electron tunneling may be addressed following the Born-Oppenheimer scheme: A partial tunneling current, $j\left(q_{k}\right)$, at fixed position $q_{k}$ of the nuclei constituting the slow vibrational subsystem may first be calculated. This current is then averaged over the distribution of the coordinates $q_{k}$ in the double-well potential formed by the adiabatic free energy surface $U_{\text {ad }}\left(q_{k}\right)^{39,40,46,48-50}$

$$
U_{\mathrm{ad}}\left(\left\{q_{k}\right\}\right)=E\left(\left\{q_{k}\right\}\right)+\frac{1}{2} \sum_{k} \hbar \omega_{k} q_{k}^{2},
$$

where $E\left(\left\{q_{k}\right\}\right)$ is the ground state energy of the electronic subsystem including the electrons of both electrodes and the electrons in the valence orbital of the bridge group.

The dependence of $E\left(\left\{q_{k}\right\}\right)$ on the vibrational coordinates is due to that of the electronic energy level in the redox group

$\varepsilon_{\mathrm{B}}\left(\left\{q_{k}\right\}\right)=\varepsilon_{\mathrm{B}}-\sum_{k} \gamma_{k} q_{k}+e\left[\varphi_{L}-\psi\left(z ; \varphi_{L}-\varphi_{L}^{\mathrm{pzc}} ; \varphi_{R}-\varphi_{R}^{\mathrm{pzc}}\right)\right]$,

where $\gamma_{k}$ are the coupling constants.

Since Eq. (B2) (and hence the energy $E\left(\left\{q_{k}\right\}\right)$ ) involves the vibrational coordinates only in the combination $\Sigma_{k} \gamma_{k} q_{k}$, a single dimensionless coordinate may be introduced

$$
q=\sum_{k} \gamma_{k} q_{k} / 2 E_{r}, \quad E_{r}=\frac{1}{2} \sum_{k} \frac{\gamma_{k}^{2}}{\hbar \omega_{k}} .
$$

The averaging over the vibrational coordinates may be then reduced to ${ }^{40}$

$$
j\left(\Delta_{L}, \Delta_{R}, \eta, V\right)=\frac{\int_{-\infty}^{\infty} j\left(q, \Delta_{L}, \Delta_{R}, \eta, V\right) \exp \left[-U_{\mathrm{ad}}\left(q, \Delta_{L}, \Delta_{R}, \eta, V\right) / k_{\mathrm{B}} T\right] \mathrm{d} q}{\int_{-\infty}^{\infty} \exp \left[-U_{\mathrm{ad}}\left(q, \Delta_{L}, \Delta_{R}, \eta, V\right) / k_{\mathrm{B}} T\right] \mathrm{d} q},
$$

where

$$
\begin{aligned}
U_{\mathrm{ad}}\left(q, \Delta_{L}, \Delta_{R}, \eta, V\right)= & \varepsilon_{\mathrm{B}}(q) n_{L}(q)+\frac{\Delta_{L}}{2 \pi} \ln \left[\varepsilon_{\mathrm{B}}^{2}(q)+\Delta^{2}\right] \\
& +\left[\varepsilon_{\mathrm{B}}(q)+e V\right] n_{R}(q)+\frac{\Delta_{\text {tip }}}{2 \pi} \ln \left\{\left[\varepsilon_{\mathrm{B}}(q)\right.\right. \\
& \left.+e V]^{2}+\Delta^{2}\right\}+q^{2},
\end{aligned}
$$

$$
\begin{aligned}
& j\left(q, \Delta_{L}, \Delta_{t R}, \eta, V\right)= \frac{2 e \Delta_{L} \Delta_{t R}}{\pi \hbar \Delta}\left[\arctan \frac{e V+\varepsilon_{\mathrm{B}}(q, \eta, V)}{\Delta}\right. \\
&\left.-\arctan \frac{\varepsilon_{\mathrm{B}}(q, \eta, V)}{\Delta}\right], \\
& n_{L}(q)=\frac{\Delta_{L}}{\Delta}\left[\frac{1}{2}-\frac{1}{\pi} \arctan \frac{\varepsilon_{\mathrm{B}}(q)}{\Delta}\right],
\end{aligned}
$$




$$
n_{R}(q)=\frac{\Delta_{R}}{\Delta}\left[\frac{1}{2}-\frac{1}{\pi} \arctan \frac{\varepsilon_{\mathrm{B}}(q)+e V}{\Delta}\right] .
$$

$\Delta_{L}$ and $\Delta_{R}$ are here the widths of the electronic energy level of the redox group due to the interaction with the corresponding electrode $\left(\Delta=\Delta_{L}+\Delta_{R}\right)$ and

$$
\varepsilon_{\mathrm{B}}(q, \eta, V)=1-2 q-\xi e \eta-\gamma e V .
$$

All energies are measured in units of the reorganization energy and $\varepsilon_{B}(q, \eta, V)$ is counted from $\varepsilon_{F L}$.

The overpotential and bias voltage are the same as in Eqs. (12) and (13).

An approximate equation is obtained at small values of the overpotential and bias voltage if approximate expressions for the rate constants of the adiabatic transitions [similar to Eqs. (A8) and (A9) with the electron transmission coefficients equal to 1] are used in Eq. (25). It is similar to Eq. (20) with

$$
\begin{aligned}
& j_{0}=e n \frac{\omega}{2 \pi} \exp \left(-\bar{E}_{r} / 4 k_{\mathrm{B}} T\right), \\
& a=\frac{E_{r R}-E_{r L}}{8 k_{\mathrm{B}} T}, \quad \bar{E}_{r}=\frac{1}{2}\left(E_{r L}+E_{r R}\right) .
\end{aligned}
$$

${ }^{1}$ R. R. Dogonadze, J. Ulstrup, and Yu. I. Kharkats, Dokl. Akad. Nauk SSSR 207, 640 (1972).

${ }^{2}$ R. R. Dogonadze, J. Ulstrup, and Yu. I. Kharkats, J. Electroanal. Chem. 39, 47 (1972).

${ }^{3}$ R. R. Dogonadze, J. Ulstrup, and Yu. I. Kharkats, J. Theor. Biol. 259, 279 (1973).

${ }^{4}$ Yu. I. Kharkats, Elektrokhimiya 8, 1300 (1972).

${ }^{5}$ Yu. I. Kharkats, A. K. Madumarov, and M. A. Vorotyntsev, J. Chem. Soc., Faraday Trans. 2 70, 1578 (1974).

${ }^{6}$ A. M. Kuznetsov and Yu. I. Kharkats, Elektrokhimiya 12, 1277 (1976).

${ }^{7}$ A. M. Kuznetsov and Yu. I. Kharkats, Elektrokhimiya 13, 1498 (1977).

${ }^{8}$ A. M. Kuznetsov and J. Ulstrup, Chem. Phys. 157, 25 (1991).

${ }^{9}$ H. M. McConnell, J. Chem. Phys. 35, 508 (1961).

${ }^{10}$ A. M. Kuznetsov and J. Ulstrup, J. Chem. Phys. 75, 2047 (1981).

${ }^{11}$ A. M. Kuznetsov, P. Sommer-Larsen, and J. Ulstrup, Surf. Sci. 275, 52 (1992)

${ }^{12}$ A. M. Kuznetsov and J. Ulstrup, Surf. Coat. Technol. 67, 193 (1994).

${ }^{13}$ E. P. Friis, Yu. I. Kharkats, A. M. Kuznetsov, and J. Ulstrup, J. Phys. Chem. A 102, 7851 (1998).

${ }^{14}$ R. R. Dogonadze and A. M. Kuznetsov, Prog. Surf. Sci. 6, 1 (1975).

${ }^{15}$ A. M. Kuznetsov, Charge Transfer in Physics, Chemistry and Biology (Gordon \& Breach, Reading, MA, 1995).
${ }^{16}$ J. Koch and F. von Oppen, Phys. Rev. Lett. 94, 206804 (2005).

${ }^{17}$ J. Koch, F. von Oppen, and A. V. Andreev, Phys. Rev. B 74, 205438 (2006).

${ }^{18}$ W. Schmickler, Surf. Sci. 295, 43 (1993).

${ }^{19}$ W. Schmickler, Surf. Sci. 335, 416 (1995).

${ }^{20}$ N. J. Tao, Phys. Rev. Lett. 76, 4066 (1996).

${ }^{21}$ W. Schmickler and N. Tao, Electrochim. Acta 42, 2809 (1997).

${ }^{22}$ J. E. T. Andersen, A. A. Kornyshev, A. M. Kuznetsov, P. Moeller, and J. Ulstrup, Elektrokhimiya 31, 984 (1995).

${ }^{23}$ A. M. Kuznetsov and J. Ulstrup, J. Phys. Chem. A 104, 11531 (2000).

${ }^{24}$ A. M. Kuznetsov and J. Ulstrup, Probe Microsc. 2, 187 (2001).

${ }^{25}$ H. Sumi, Chem. Phys. 222, 269 (1997).

${ }^{26}$ H. Sumi, J. Phys. Chem. B 102, 1833 (1998).

${ }^{27}$ H. Sumi, Y. Hori, and K. Mukai, J. Electroanal. Chem. 592, 46 (2006).

${ }^{28}$ J.-D. Zhang, Q. Chi, A. M. Kuznetsov, A. G. Hansen, H. Wackerbarth, H. E. M. Christensen, J. E. T. Andersen, and J. Ulstrup, J. Phys. Chem. B 106, 1131 (2002)

${ }^{29}$ J.-D. Zhang, A. M. Kuznetsov, and J. Ulstrup, J. Electroanal. Chem. 541, 133 (2003).

${ }^{30}$ A. M. Kuznetsov and J. Ulstrup, Elektrokhimiya 31, 244 (1995).

${ }^{31}$ T. Albrecht, K. Moth-Poulsen, J. B. Christensen, A. Guckian, T. Bjornholm, J. G. Vos, and J. Ulstrup, Faraday Discuss. 131, 265 (2005).

${ }^{32}$ T. Albrecht, A. Guckian, A. M. Kuznetsov, J. G. Vos, and J. Ulstrup, J. Am. Chem. Soc. 128, 1732 (2006).

${ }^{33}$ A. Alessandrini, S. Corni, and P. Facci, Phys. Chem. Chem. Phys. 8, 4383 (2006)

${ }^{34}$ Q. Chi, J.-D. Zhang, P. S. Jensen, H. E. M. Christensen, and J. Ulstrup, Faraday Discuss. 131, 181 (2005).

${ }^{35}$ Z. Li, B. Han, G. Meszaros, I. Pobelov, Th. Wandlowski, A. Blaszczyk, and M. Mayor, Faraday Discuss. 131, 121 (2006).

${ }^{36}$ F. Chen, J. He, C. Nuckolls, T. Roberts, J. E. Clare, and S. Lindsay, Nano Lett. 5, 503 (2005).

${ }^{37}$ X. Xiao, L. A. Nagahara, A. M. Rawlett, and N. Tao, J. Am. Chem. Soc. 127, 9235 (2005)

${ }^{38}$ I. Visoly-Fisher, K. Daie, Y. Terazono et al., Proc. Natl. Acad. Sci. U.S.A. 103, 8686 (2006)

${ }^{39}$ A. M. Kuznetsov and W. Schmickler, Chem. Phys. 282, 371 (2002).

${ }^{40}$ I. G. Medvedev, J. Electroanal. Chem. 600, 151 (2007).

${ }^{41}$ A. A. Kornyshev and A. M. Kuznetsov, ChemPhysChem 7, 1 (2006).

${ }^{42}$ A. A. Kornyshev and A. M. Kuznetsov, Chem. Phys. 324, 276 (2006).

${ }^{43}$ A. A. Kornyshev, A. M. Kuznetsov, and J. Ulstrup, Proc. Natl. Acad. Sci. U.S.A. 103, 6799 (2006)

${ }^{44}$ A. A. Kornyshev and A. M. Kuznetsov, Electrochem. Commun. 8, 679 (2006).

${ }^{45}$ D. V. Averin and Y. V. Nazarov, Phys. Rev. Lett. 65, 2446 (1990).

${ }^{46}$ A. M. Kuznetsov and I. G. Medvedev, Electrochem. Commun. 9, 6 (2007).

${ }^{47}$ A. A. Kornyshev, A. M. Kuznetsov, and J. Ulstrup, ChemPhysChem 6, 583 (2005).

${ }^{48}$ I. G. Medvedev, Russ. J. Electrochem. 39, 44 (2003).

${ }^{49}$ I. G. Medvedev, Russ. J. Electrochem. 41, 227 (2005).

${ }^{50}$ I. G. Medvedev, Russ. J. Electrochem. 41, 368 (2005). 